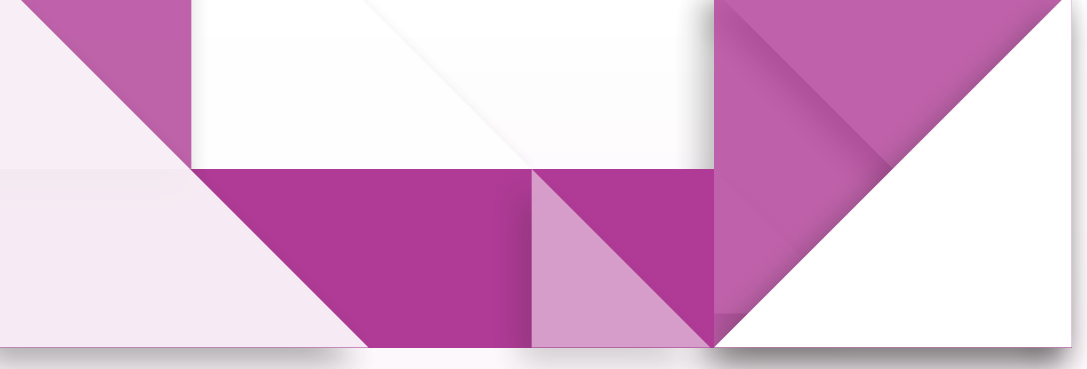

\title{
Concepciones de biodiversidad y prácticas de cuidado de la vida desde una perspectiva cultural
}

- Conceptions of Biodiversity and Practices of Care for Life from a Cultural Perspective

- Concepções de biodiversidade e práticas de cuidado da vida a partir de uma perspectiva cultural

\section{Resumen}

Este artículo presenta los resultados de un trabajo de investigación realizado con estudiantes de la Licenciatura en Biología y pobladores de la etnia vitoto-muruy de la Chorrera-Amazonas, cuyo propósito central es reconocer las concepciones de biodiversidad y las prácticas de cuidado, que aporten a la discusión respecto la memoria biocultural del país y a las reflexiones necesarias para la formación de profesores de biología. Se siguió una metodología cualitativa, con un enfoque interpretativo-hermenéutico. Se utilizó el diario de campo, la observación participante, entrevistas semiestructuradas, entrevistas en profundidad, registro visual e identificación de actores sociales como técnicas y herramientas, las cuales permitieron establecer un diálogo abierto respecto a las formas de concebir la biodiversidad, las prácticas de cuidado y su articulación con el territorio. Dentro de estos resultados, se destaca la importancia de la cultura y las maneras de significar la biodiversidad por parte de los grupos sociales y la forma de convivir con las otras formas de existencia en el territorio; así como las prácticas de cuidado, que se expresan en las formas de comprender el mundo, de habitarlo, de producirlo y de cuidarlo.

Palabras clave

concepciones; biodiversidad; formación de profesores; cultura; prácticas de cuidado

\section{Abstract}

María Rocío Pérez Mesa* Licenciada en Biología. Especialista
en Educación Ambiental, Magíster en
Educación, doctora en Educación.
Profesora de planta de la Universidad
Pedagógica Nacional. Artículo resultado
de investigación.

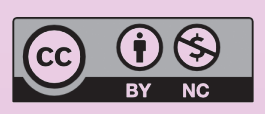


This paper presents the results of a research work carried out with students from the Bachelor of Arts in Biology and people of Witoto-Muruy ethnicity from the town of La Chorrera, Department of Amazonas, the main goal of which is to recognize the conceptions of biodiversity and the care practices that contribute to the discussion regarding the biocultural memory of the country and to the reflections required for the training of biology teachers. A qualitative method with an interpretive-hermeneutic approach was used. Field diaries, participant observation, semi-structured interviews, in-depth interviews, visual records and identification of social actors were used as techniques and tools, which enabled the establishment of an open dialogue about the ways of conceiving biodiversity, care practices and their articulation with the territory. Among these results, it is worth highlighting the importance of culture and the ways that social groups give meaning to biodiversity and how to coexist with other forms of existence in the territory, as well as care practices, which are expressed in the ways of understanding the world, inhabiting it, producing it, and caring for it.

Keywords

conceptions; biodiversity; teacher training; culture; care practices

\section{Resumo}

Este artigo apresenta os resultados de uma pesquisa realizada com estudantes de Licenciatura em Biologia e moradores da etnia Uitoto-Muruy de La Chorrera-Amazonas, cujo propósito principal foi reconhecer os conceitos de biodiversidade e as práticas de cuidado que pudessem contribuir para a discussão sobre a memória biocultural do país e as reflexões necessárias para a formação de professores de biologia. Uma metodologia qualitativa foi seguida, com uma abordagem hermenêutica interpretativa. Foram utilizados diários de campo, observações participantes, entrevistas semi-estruturadas, entrevistas em profundidade, registro visual e identificação de atores sociais como técnicas e ferramentas, o que permitiu estabelecer um diálogo aberto sobre as formas de conceber a biodiversidade, práticas de cuidado e sua articulação com o território. Dentro desses resultados, destacamos a importância da cultura e das formas de significar a biodiversidade por parte dos grupos sociais, a convivência com outras formas de existência no território, bem como as práticas de cuidado, que se expressam nos modos de entender o mundo, de habitá-lo, de produzi-lo e de cuidar dele.

Palavras-chave

concepções; biodiversidade; formação dos docentes; cultura; práticas no cuidado 


\section{Introducción}

La investigación corresponde a un trabajo realizado en el marco de la tesis doctoral sobre concepciones de biodiversidad desde la perspectiva de la diversidad cultural, con profesores en formación inicial de la Licenciatura en Biología, la cual se desarrolla en el contexto de la Chorrera (Amazonas) y en la ciudad de Bogotá. Particularmente, el presente artículo hace énfasis en el trabajo que se adelantó en el contexto de la Chorrera (Amazonas), con pobladores y futuros profesores de biología, pertenecientes a la etnia vitoto-muruy.

La Chorrera (Amazonas) se encuentra ubicada en la región del Amazonas y es reconocida a nivel mundial por la diversidad biológica y cultural, conformada por tres complejos socioecológicos: áreas de selva húmeda del piedemonte y andes amazónicos, áreas de selva húmeda de la llanura amazónica y el área del trapecio amazónico. Sin embargo, la transformación de la cuenca amazónica por actividades extractivas, principalmente de caucho, para mercados internacionales a finales del siglo xIx e inicios del siglo Xx, impactaron la base biofísica y cultural de esta región, al propiciar uno de los mayores etnocidios en la historia del país (Palacio, 2001; Urbina, 2010).

En tal sentido, advierte una problemática de fondo que se empieza a visibilizar en el mundo contemporáneo, como es la pérdida de diversidad biológica y cultural, al igual que la erosión de los conocimientos ancestrales (Maffi, 2005; Toledo y Barrera-Bassols, 2008), agenciados por procesos hegemónicos de poder, asimetrías de conocimientos y de prácticas insostenibles del proyecto civilizatorio, que han llevado al límite los procesos de explotación de la naturaleza y su mercantilización, a la par del marginamiento de los grupos minoritarios.
Lo anterior requiere una reflexión desde la educación y la formación de licenciados en biología, como parte fundamental de las realidades de país (Bravo, 2015), para avanzar hacia una educación que reconozca las diversidades y los diálogos necesarios desde las diferencias, como una sociedad que se piensa pluriétnica y multicultural, en uno de los países más biodiversos del planeta. En tal sentido, se plantea como pregunta: ¿̇qué relaciones emergen entre las concepciones de biodiversidad y las prácticas culturales de estudiantes de la Licenciatura en Biología y pobladores de la etnia vitoto-muruy en la Chorrera (Amazonas)?

De esta manera, la investigación presenta resultados y discusiones respecto a las concepciones de biodiversidad, como una aproximación a su sistema de conocimientos y prácticas, que les ha posibilitado resistir y abrirse paso, para hacer de esta región un lugar biodiverso desde un convivir y un saber habitar. Por tanto, estos conocimientos, permiten esbozar elementos de relación, comprensión y proyección, para que sean discutidos en los procesos de formación de profesores de biología, en un ejercicio de reconocimiento a las diferencias, de posibilitar el tejido de puentes entre conocimientos (Melo, 2017), desde un pluralismo epistemológico (Olive, 2009) y una ética del cuidado de la vida, que también redunda en una construcción de un país en paz.

\section{Origen del término}

El origen del término biodiversidad aparece en el ámbito político (Ghilarov, 1996) y se le atribuye una estrecha relación con el ambiente, la tecnología y la política, más que con la misma ciencia. Sin embargo, fue asimilado rápidamente por los científicos como una vía para el fortalecimiento de investigaciones y la obtención de financiamiento en las áreas de sistemática y ecología. De acuerdo con Jeffries 
(1997), durante la década de 1980, Lovejoy y Norse y McManus produjeron documentos para el Gobierno estadounidense, en los que incorporan la expresión diversidad biológica (Pérez, 2013).

Así, la diversidad biológica como expresión acuñada por Lovejoy (1980), sería objeto de mayores refinamientos, en este proceso de construcción; fue Walter Rosen (1985), citado en Tacaks (1996, p. 37), quien propone el neologismo biodiversidad, en una reunión previa del Foro Nacional sobre Biodiversidad, realizado en Washington, auspiciado por la Academia Nacional de Ciencias y el Instituto Smithsoniano, término popularizado por Wilson al editar las memorias del evento bajo el título "Biodiversidad", con importantes repercusiones desde la década de 1990 hasta el presente.

En el marco de la Cumbre de Río (1992), se hace un reconocimiento oficial por parte de representantes de más de noventa países sobre la importancia de la biodiversidad y se proporciona una definición al mundo como se referencia:

[...] la variabilidad de organismos vivos de todo origen comprendido, entre otros, los ecosistemas terrestres, acuáticos y los complejos ecológicos de los cuales hacen parte; ello comprende la diversidad en el seno de las especies y entre las especies así como la de los ecosistemas. (CDB, 1992).

En tal sentido, Sarkar (2008) plantea que la biodiversidad, al referirse a todas las entidades biológicas, se convierte en toda la biología, aspecto que conlleva serias implicaciones en el momento de concretar acciones para la conservación, dado que todo el mundo viviente se convertiría en un objetivo de conservación (Sarkar, 2005, p. 180), mientras que Solbrig (1991) define la biodiversidad como la propiedad de los seres vivos de ser diferentes entre sí, por lo que estima que no es una entidad sino una propiedad. De igual manera, se sigue enriqueciendo la discusión desde aportes de la ecología en relación con la diversidad funcional, al conferirle un papel activo a la biodiversidad, aspectos que pueden ser ampliados en Pérez (2013).

\section{Emergencia de lo diverso: concepciones de biodiversidad}

Abordar las concepciones de biodiversidad desde una perspectiva cultural supone el reconocimiento de lo diverso, de lo diferenciado, de donde emerge el sujeto situado, perteneciente a un colectivo, cuyos conocimientos son proporcionados mediante ideas, palabras e imágenes, como marcos de interpretación de realidades asociadas con la biodiversidad. En estos términos, las concepciones de biodiversidad, plantean una pluralidad epistémica (Olivé, 2009) y ontológica, así como una dimensión de lo ético, al considerar otras lecturas de las relaciones entre los grupos culturales y la biodiversidad como nuevo énfasis de la naturaleza.

De acuerdo con estudios arqueológicos, parte de la biodiversidad actual es fruto de la manipulación e intervención de los ecosistemas y especies que diferentes culturas ejercían sobre su entorno (Ulloa, 2004). La llamada vida silvestre 
encontrada por los colonizadores europeos, había sido el resultado del manejo de la tierra por los nativos americanos (Cronon, 1983 y Denevan, 1992; en Takacs, 1996, p. 42), aspecto que no se aparta de los grupos indígenas del Amazonas, quienes han dado manejo a la naturaleza desde tiempos milenarios (Toledo y Barrera-Bassols, 2008).

La biodiversidad como nuevo énfasis de la naturaleza, si bien es un término occidental, ha sido objeto de negociación y transformación en diversos grupos étnicos, de acuerdo con sus formas de conocimiento y sus prácticas culturales. A este respecto, se destacan las concepciones de biodiversidad desde las etnias, las cuales, pueden ser interpretadas en las culturas orales sustentadas en una filosofía basada en un saber práxico por medio del mito, donde la palabra tiene un lugar privilegiado como gestora de saber y recuerdo, abierto al cambio (Montoya, 1994; Rozo, 2005).

Las diferentes formas de concebir la biodiversidad posicionan otras lógicas que señalan el vínculo de lo humano y la naturaleza, de manera que el ser humano no capta la realidad como algo ajeno, sino la hace copresente como un momento mismo de su ser junto, de la originariedad holística (Estermann, 1998, p. 92). Así, para diversos pueblos como el wayuu, todos los componentes de la naturaleza están dotados de vida "piedras, arroyos, vientos y la tierra, todo tiene espíritu y por tanto, todo absolutamente es considerado como biodiversidad" (Ramírez, 2005, p. 40).

\section{Biocultura}

En el mundo contemporáneo la biodiversidad es asumida por algunos académicos como un nuevo énfasis de la naturaleza (Ulloa, 2011), además de ser considerada simultáneamente real, colectiva y discursiva (Escobar, 1999). Esta diversidad biocultural, reconoce el estre- cho vínculo entre lo humano y lo no humano, como real, y como una producción discursiva y de significación, que fuera invisibilizada durante siglos (Toledo y Barrera-Bassols, 2008). Para Maffi (2005), el campo de la diversidad biocultural durante la última década se ha planteado como un área de investigación transdisciplinaria relacionada con los vínculos entre la diversidad lingüística, cultural y biológica del mundo, asumiéndolas como manifestaciones de la diversidad de la vida. Estas nuevas reinvenciones de la naturaleza y del llamado Tercer Mundo, incorporan otras formas de aproximarse al conocimiento de los "otros" para entender cómo las culturas de tradición oral han logrado sobrevivir y, a su vez, conservar la biodiversidad.

Por tanto, se requiere de un trabajo que permita reconocer estas realidades, y problematizarlas no solo desde la antropología, la historia, la sociología y la ecología política, pues también debe ser objeto de debate por parte de la educación en ciencias y la formación de profesores, como visiones alternas a las miradas clásicas, que requieren ser abordadas para favorecer un diálogo fértil de saberes en la comprensión de los diversos significados de la biodiversidad y la cultura que aporten en la construcción de propuestas educativas con un sentido de realidad.

\section{Metodología}

La metodología empleada es de corte cualitativo desde el enfoque interpretativo, y permite hacer un estudio epistemológico en torno a las formas de construir el conocimiento sustentado en las relaciones entre el sujeto cognoscente, el sujeto conocido y el contexto, que busca aproximarse al universo interpretativo del otro (Geertz, 1987; Molina, 2005; Vasilachis, 2006). Para ello se emplea el concepto semiótico de cultura (Geertz, 1987, 1996), 
los procesos de significación (Bruner, 1984, 1988, 2006; Plantin, 2014) y la propuesta de la cultura en términos adjetivos (García Canclini, 2004). Estos referentes permiten comprender las formas de enunciación y significación desde el lugar de la cultura y la elaboración de sentido, para referirse a la biodiversidad como nuevo énfasis de la naturaleza, desde sus marcos de referencia (Geertz, 1987), por la relación entre el sentido y la referencia (Molina, 2012), desde la cultura y la polisemia que el término entraña.

La perspectiva que se asume en la investigación toma en consideración la cultura, el contexto cultural, el encuentro entre diferentes y la diversidad cultural en términos adjetivos (García Canclini, 2004), como expresión de las sociedades contemporáneas y la interculturalidad, que hace parte de la configuración histórica del país. Así, la cultura como un sistema de significados, implica tener en cuenta las formas de enunciar y significar la realidad por parte de los docentes en formación, donde lo cultural se constituye en un recurso heurístico para hablar de la diferencia, de los contrastes y las comparaciones cuando los grupos y los sujetos entran en relaciones de intercambio que comprenden la negociación de los significados y el cruce de fronteras, de acuerdo con las experiencias y los contextos desde el reconocimiento de la diferencia y la otredad.

El estudio de las concepciones implica referentes contextuales y socioculturales que inciden en su configuración, en un despliegue de diversidad y complejidad que hacen parte de la investigación. De modo que se reconocen las formas de enunciación y significación de la biodiversidad, al igual que las prácticas de cuidado por parte de las voces de futuros profesores de biología pertenecientes a la etnia vitoto-muruy y los diálogos con pobladores de la Chorrera (Amazonas) $y$, en tal sentido, todo ello es posible gracias al apoyo brindado por Azicatch y el consentimiento informado de los participantes quienes siempre manifestaron su profundo interés y destacada participación.

De esta forma, la investigación se enmarca en una perspectiva emergente de las concepciones de los profesores en formación inicial de ciencias en clave cultural, pues como señalan Molina et al. (2014, p. 18), la mayoría de investigaciones de concepciones han estado orientadas al estudio de la naturaleza del conocimiento científico y el aprendizaje, pero son incipientes las que han caracterizado la relación con la diversidad cultural, así como sus implicaciones en la enseñanza de las ciencias y la formación inicial de profesores, desde la otredad, la diferencia, la diversidad y el contexto cultural.

\section{Resultados y análisis}

En el contexto de la cultura uitoto-muruy es importante considerar que su sistema de conocimiento se sustenta en el pensamiento-la palabra y la obra; desde allí se construye la realidad y en su producción colectiva se entretejen el mundo espiritual, lo humano y la naturaleza como unidad. El pensamiento es discurso 
y práctica, que se transmite en el colectivo, el cual parte de lo mítico, para hablar de un interrogante que ha hecho parte de las diferentes culturas y es la pregunta por el origen de la vida y su diversidad (figura 1).

\section{El origen de la vida y su diversidad}

Puedo afirmar que la vida es una sola para la cultura y su relación tiene un mismo origen, el origen del hombre, el origen de los clanes, de cada una de las especies, tiene un solo origen tiene un solo principio, entonces este principio obedece precisamente a lo que en nuestra cultura está dado prácticamente por el orden de la creación, porque la expresión que se dice (Tan non Jagtytcomuya), o sea la expresión es que todo comenzó todo inició de la nada.

En la narración en el (Zomarafue), dice que primero tiene que ver con una forma de traslado, de superación que va cogiendo esa esencia de vivir.

En comuya buinaima está presente la palabra de multiplicarse de abundancia, donde prácticamente se consolida el proceso de formación de la vida, entonces para nosotros la vida es una sola. Nuestra cultura por eso se dice todo lo hago o lo hice con la palabra (nana uñuauyay do cuefi moca), con la palabra del cuidado y la protección de la vida io vine haciendo, entonces uno da a entender de que la vida es una sola. (Faredkade. Sabedor Uitoto-Muruy, 2013).

Figura 1. El origen de la vida y su diversidad según sabedor vitoto-muruy

Fuente: elaboración propia.

Al hablar de la vida y su diversidad, se remite al origen que convoca el acto creador, donde la existencia primera es la nada y el sueño es el punto de partida como instancia del pensamiento para crear, a través de la palabra, el mundo como realidad. Lo soñado es el mundo de los vitoto-muruy, plantas, animales, colores y aromas del paisaje forman parte del pensamiento que se vuelve realidad y a través de la palabra comuya buinaima, la palabra de multiplicarse, de abundancia, se consolida el proceso de formación de la vida (Farekatde, sabedor vitoto-muruy, 2013). La palabra representa la historia misma de esta cultura, sus formas de conocer y aprender el mundo, en ella se condensa el origen del mundo y de la vida.

La palabra que se transmite a través de la tradición permite una forma de adquisición del saber y de mantener la memoria acerca del origen como unidad ontológica que se hace pública, para responder a la pregunta común a todas las culturas acerca del origen de la vida y sus diversas formas, con las diferentes elaboraciones inherentes a cada cultura y que en el caso de la etnia uitoto-muruy, se establecen desde la palabra de vida.

En todo ese conjunto de mitos y leyendas lo que hace todo que desde lo espiritual se vuelva materia y en esa búsqueda aparecieron animales, aparecieron peces de todo, (kafkomuya Jagłyf) con ese aire se vino a transformar y ahí apareció todo.

(Gil Jairo, 2013).

En el mito de origen se visibiliza el acto creador como un proceso que busca dar respuesta al origen y existencia del humano y de las demás formas de vida, desde el plano espiritual y su transformación en materia a través del aire de vida Ta+noJagłył. Las diferentes entidades no humanas están dotadas de espíritu y en la búsqueda del hombre, aparecen modeladas las diferentes formas de vida que dan cuenta de la diversidad de seres existentes, de manera que puede resolver una de las características de la vida como crear formas estables (Pitrou, Valverde y Neurath, 2011 , p. 32), además de compartir un mismo origen como lo expresa Gil Farekatde, sabedor vitoto-muruy (2013).

\section{El sistema de conocimiento y los escenarios de aprendizaje}

El sistema de conocimiento parte del mito de origen y la ley de vida que se transmite al colectivo desde los cuatro escenarios de aprendizaje como son la maloca, el río, la selva y la chagra, los cuales expresan una profunda interrelación entre la naturaleza-lo humano y lo espiritual. De esta forma, la vida tanto en lo individual como en lo colectivo, se organiza desde los mitos como sabiduría del pueblo vitoto configurado por diversos clanes. 


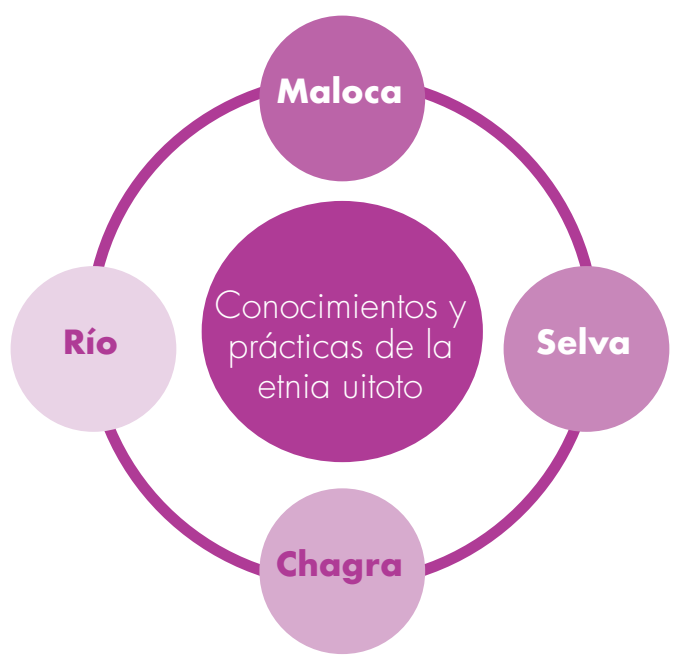

Figura 2. Escenarios de aprendizaje de la cultura vitoto-muruy

Fuente: elaboración propia.

Para la comunidad, y en particular para los futuros licenciados, estos escenarios de aprendizaje del conocimiento propio permiten un aprendizaje para toda la vida, el cual se transmite a todos los miembros, a lo largo de sus vidas. De esta forma, este pueblo originario ha logrado proyectarse hasta la actualidad, en su organización, pensamiento y acción, dadas sus fortalezas y el poder de una educación propia que ha sido sostenida por sus sabedores y demás miembros, con diferentes formas de participación de un conocimiento que se teje y se desteje desde lo colectivo. De esta manera, se explica el mundo desde lo mítico, y a través de diferentes rituales se busca armonizar las relaciones entre la naturaleza-lo humano y lo espiritual.

\section{Concepciones de biodiversidad}

Las formas de enunciar y significar la biodiversidad desde el lugar de la cultura, muestran la importancia que para los participantes representan la vida y sus diversas manifestaciones en el contexto local. A continuación se explicitan las formas de nombrar la diversidad de la vida por parte de los futuros licenciados en biología de la etnia vitoto.

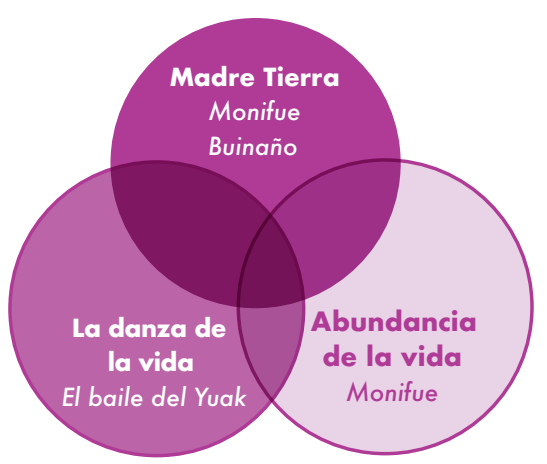

Figura 3. Concepciones de biodiversidad desde un nombrar propio Fuente: elaboración propia. 
Estas concepciones permiten afirmar la diferencia y plantear un término más incluyente y lleno de significado y sentido dentro de su contexto cultural, al mantener la coherencia de la unidad ontológica (naturaleza-humanidad-espiritualidad), su epistemología (el sistema de conocimiento que la crea) y su ética del cuidado, que confronta el concepto occidental.

\begin{tabular}{|c|c|c|}
\hline $\begin{array}{l}\text { Madre Tierra. } \\
\text { Monifue Buinaño } \\
\text { (Pensamiento Uitoto) }\end{array}$ & $\begin{array}{l}\text { Abundancia de la } \\
\text { vida Monifue: un } \\
\text { saber cuidar la vida } \\
\text { (La palabra que regula) }\end{array}$ & $\begin{array}{l}\text { La Danza de la vida } \\
\text { El Baile de Yuak+ } \\
\text { (pensamiento y palabra } \\
\text { que se lleva a la obra) }\end{array}$ \\
\hline $\begin{array}{l}\text { Estamos contentos } \\
\text { con los animales, } \\
\text { estamos contentos } \\
\text { con las plantas, nos } \\
\text { provee muchas } \\
\text { cosas, por lo que } \\
\text { digo biodiversidad } \\
\text { para nosotros } \\
\text { no sería solo del } \\
\text { término, sino } \\
\text { seria otra cosa, la } \\
\text { podríamos definir } \\
\text { desde un concepto } \\
\text { propio[...] les la } \\
\text { Madre Tierra!, } \\
\text { la Madre Tierra, } \\
\text { porque es la que } \\
\text { engendra todo, } \\
\text { (E.I Francisco) }\end{array}$ & $\begin{array}{l}\text { Monifue es todo la } \\
\text { naturaleza todo, } \\
\text { hasta el mismo } \\
\text { hombre, ahí está, } \\
\text { pero entonces } \\
\text { ya para que sea } \\
\text { monifue o sea que } \\
\text { ya se pueda tener } \\
\text { vida, que le ayude } \\
\text { al hombre, todo } \\
\text { eso está regulado } \\
\text { es por la palabra, } \\
\text { ahí está la clave de } \\
\text { esta palabra, [...] } \\
\text { pueden ser frutales, } \\
\text { pueden ser peces, } \\
\text { ser animales, todo } \\
\text { es monifue todo es } \\
\text { monifue, itodo! } \\
\text { (E.3. Gil.Jairo) }\end{array}$ & $\begin{array}{l}\text { El baile del Yuak+ } \\
\text { que para nosotros } \\
\text { es el baile de la } \\
\text { naturaleza, va } \\
\text { relacionado con } \\
\text { todo lo que existe en } \\
\text { este mundo, lo que } \\
\text { vemos acá, los seres } \\
\text { del agua. y los seres } \\
\text { de la tierra, los que } \\
\text { vuelan y lo espiritual, } \\
\text { con la abundancia, } \\
\text { el baile del Yuak+ } \\
\text { armoniza todo. } \\
\text { (E.2 Fidelia) }\end{array}$ \\
\hline
\end{tabular}

Figura 4. Formas de nombrar y significar la biodiversidad desde la cultura

Fuente: elaboración propia con base en lo expresado por los participantes.

Cada una de las formas de nombrar y significar la biodiversidad muestra la amplitud y capacidad de relacionar con el pensamiento mítico de la Madre Tierra y del Monifue, como formas explicativas de la vida y su diversidad, en las cuales es posible apreciar una interrelación entre lo humano, la naturaleza y lo espiritual. La Madre Tierra es sagrada para la etnia vitoto, porque es la que engendra, la que da vida $y$, por tanto, no hay un sentido de explotación sostenida o privatización ni mercantilización, como acontece en la cultura hegemónica.

La palabra monifue simboliza todas las formas de existencia, todo lo que tiene vida, ello incluye también el agua, las rocas, el viento, entre otras entidades que hacen parte del mundo y, por tanto, deben ser reconocidas y valoradas como parte integral del territorio que se habita. El Monifue representa la abundancia, la diversidad de la vida, en la que todo está dotado de espíritu, y las diferentes entidades para que tengan vida deben estar reguladas por la palabra, que remite a un origen compartido y comprende la continuidad de las interioridades entre humanos y naturaleza (Descola, 2012), como se reafirma en los diferentes diálogos con Gil Jairo.

Si bien la categoría de biodiversidad no ha sido ajena a su formación escolar y al currículo intercultural de su formación universitaria, desde su contexto cultural se hace una apropiación ontológica del mundo y a su vez, se reconocen otras formas de concebir el mundo como las del dominio ontológico occidental que separa la vida de lo vivo (Escobar, 201 1, p. 67). De esta manera, se reconoce la diferencia ontológica que emerge entre Occidente y la forma de significar la biodiversidad, en relación con las concepciones desde nombrar y significar la diversidad de la vida en la etnia vitoto.

Por su parte, el baile del Yuak + se constituye en uno de los principales rituales de la etnia que comprende un diálogo con todas las entidades del mundo, para armonizar las relaciones y el equilibrio entre la naturaleza, lo humano y lo espiritual. El baile aparece como un modelo que vincula el espacio y el tiempo, al estar estructurado en grandes ciclos rituales además de preservar la memoria y revalidar el vínculo entre la naturaleza, lo humano y lo espiritual como un vivir comunitario. El baile del Yuak + o baile de la naturaleza, permite condensar todo aquello que significa y vincula la diversidad de la vida en todas sus manifestaciones, como una expresión de los conocimientos y las prácticas de la cultura. 
Este baile tiene un claro referente identitario desde la cultura y el clan, al ser este uno de los cuatro bailes más representativos en su configuración histórica y mítica que recuerda el origen de la diversidad y el vínculo entre la naturaleza, los humanos y lo divino, como una forma de construir la realidad; este baile es instituido por "Buinaima y su esposa Buinaiño, la Gran Madre" (Urbina, 2010, p. 39), y guarda un significado asociado con la abundancia de las cosechas y las relaciones armónicas con la naturaleza. Desde esta experiencia es posible apreciar la reiteración del todo relacionado, al vincular la palabra, el buen nombrar, el tótem, la unidad ontológica y las prácticas de cuidado, en una relación dinámica con la naturaleza dotada de vida.

\section{Prácticas de cuidado}

Los vitoto somos, 'D+oma o D+onuy-Uruk+', el hombre que cuida el conocimiento de la vida.

Azicatch (2007).

El cuidado del conocimiento para la etnia vitoto se basa en el principio de relacionalidad entre la naturaleza, lo humano y lo espiritual, al simbolizar la unidad ontológica del todo integrado. Se conoce el mundo desde la experiencia, desde los trayectos del ser que entreteje con el colectivo a través del que se comparten los significados de la cultura que le permiten configurar un conocimiento situado, al privilegiar el conocimiento del cuidado de la vida como filosofía de existencia. Se explicita, entonces, el pensamiento como discurso y como prácticas de cuidado mediante la armonización de las relaciones que se sustentan en el conocimiento como forma de producir la realidad y desde la educación propia se fortalece en los cuatro escenarios de aprendizaje que vinculan la salud, el alimento y el territorio ancestral para convivir con los demás seres, tanto del mundo tangible como intangible.

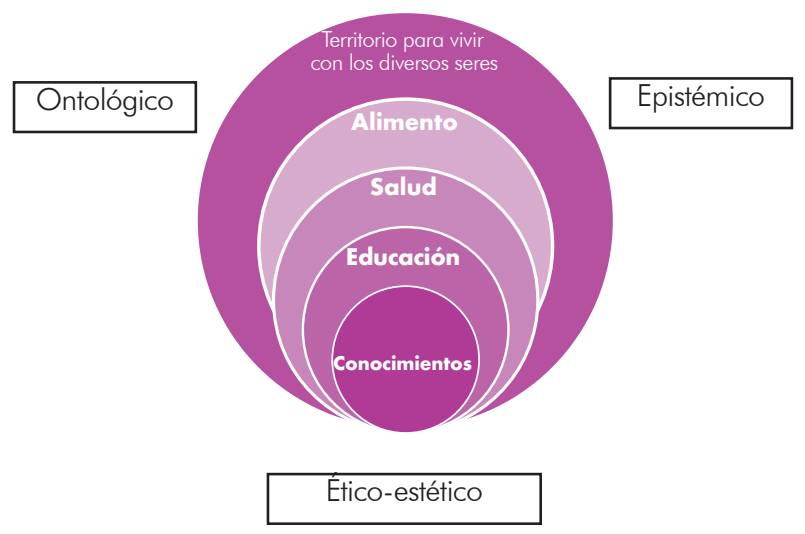

Figura 5. Expresión de las prácticas de cuidado

Fuente: elaboración propia. 


\section{Lo ontológico}

La unidad ontológica afirma la identidad cultural y supone la integralidad con el lugar que se habita, en interrelación con los otros, con la naturaleza y la espiritualidad; en tal sentido, orienta la vida misma y el orden social, como expresa el sabedor vitoto: "Los que ya hacen maloca, es para mirar la situación el análisis y la protección de la vida del orden ya social, [...] precisamente desde el Zomarafue" (Farekatde, sabedor vitoto-muruy, 2013).

La maloca, entonces, simboliza la historia de los pueblos del tabaco, la coca y la yuca dulce; condensa el conocimiento de la cultura y, a su vez, simboliza a la madre naturaleza que refleja la estructura del universo y su proceso cosmogónico (Urbina, 2010, p. 45). Allí se estrecha el vínculo entre materialidad y espiritualidad para proteger la vida, conocimiento que es orientado por el sabedor a través de complejas prácticas ceremoniales y rituales para armonizar las relaciones de la naturaleza, lo humano y lo espiritual, el cuidado de la salud, del alimento, monitorear el territorio y alinearlo con el cosmos, como se expresa en el plan de vida de Azicatch (2007).

\section{Lo epistémico}

El conocimiento se teje y se desteje en el colectivo, desde su historicidad. Como forma de producir el mundo y sostenerlo. El cuidado del conocimiento parte del reconocimiento de una historia de la vida misma, en tanto configura una realidad interrelacionada, como una forma de significar el mundo. Comprende un proyecto cultural que se recrea a través de cómo conoce el mundo el vitoto, de su ser individual y colectivo, pilares de esa relación a través de la palabra y las prácticas de cuidado, que remiten a un mismo origen y demandan unas relaciones de igualdad y respeto entre la humanidad y la naturaleza, necesarias para el mantenimiento de la vida y la permanencia como grupo.
[...] nosotros por ejemplo sabemos este sirve para tal cosa, aquello sirve para tal cosa, esas plantas y con todo eso va acompañado con la oración, que es aire de vida de abundancia [mm] o sea el soplo, lo que se habla lo que no se puede palpar, y esa es la parte fundamental porque eso va ligado con el padre creador, o sea el hombre indígena no puede decir no, que yo solo hice esto, tenemos nuestros antecesores, por eso el padre creador usa la coca y tabaco como herramienta. (Fidelia, 2013).

En esta perspectiva resulta relevante reconocer la configuración histórica del conocimiento del cuidado de la vida, sustentada en las relaciones entre la naturaleza, lo humano y lo espiritual, conocimiento que se actualiza y enriquece desde la experiencia, considerando un colectivo con unas prácticas sociales, que se ponderan al contribuir a la permanencia de la vida en sus múltiples manifestaciones y formas de significar.

El baile se constituye en la memoria viva de la etnia, al posibilitar un momento privilegiado para narrar los mitos, como expresión del conocimiento que se comparte y se teje colectivamente y que busca mantener la unidad ontológica y la armonización de las relaciones entre la naturaleza, lo humano y lo espiritual, que hace parte de la experiencia de Fidelia, hija del cacique dueño de la maloca, al participar en estas prácticas rituales y ceremoniales.

El baile como práctica epistémica, de acuerdo con Olive (2009), comprende un conocimiento sofisticado de la naturaleza, de la tradición milenaria, que corresponde al saber condensado en los mitos, las letanías, los conjuros, los cantos y las danzas, como parte del complejo sistema de conocimiento, que se comparte con el colectivo y que exhibe la capacidad de ordenar la naturaleza y el cosmos, de velar por la armonización de las 
relaciones como instancia en la que se funda su conocimiento y la abundancia que contempla el conocimiento, para el cuidado de la vida a través del alimento, la salud, la producción de la vida y la fecundidad (Urbina, 2010).

El conocimiento holístico y ecológico de la etnia reconoce la creatividad de la naturaleza y, a su vez, participa en la producción y protección de la vida a través del cuidado de la salud, el alimento, el saber convivir en un todo interrelacionado y la transmisión del conocimiento. La Chorrera, como complejo biológico-cultural, muestra el vínculo entre estas dos diversidades como una relación biocultural, que expresa un corpus de conocimiento configurado históricamente en su interacción con el río, la selva y la chagra.

\section{Lo ético y lo estético}

La diversidad de la vida representada por la madre naturaleza o madre de abundancia, como fuente de diversidad y de alimento, es valorada por la etnia al considerar la vida dotada de espíritu que se constituye en fundamento y regulación de sus prácticas. Desde el origen se plantea una relación de igualdad entre la naturaleza, lo humano y lo espiritual y en esta interacción se teje un conocimiento colectivo y se revalidan las relaciones entre humanos y no humanos a través de la armonización de las relaciones.

Nadie es superior a nadie, todo es una relación entre iguales. (Farekatde, sabedor vitoto-muruy, 2013)

Para nosotros todo lo que existe tiene también vida, que son hermanos nuestros de una u otra forma ya lo decía al principio, con lo de los tótems. (Fidelia, 2013)

La diversidad de la vida comprende el conocimiento de la vida como acontecimiento vivo, donde todo se encuentra interrelacionado. En tal sentido, emerge la otredad, al asumir la naturaleza como sujeto "dotada de espíritu", que exige un reconocimiento y también unos compromisos del vitoto, en su relacionamiento. El conocimiento para el cuidado de la vida, obedece a unas normas como el saber dietar y recibir consejo por parte de los mayores, es decir que ello implica regular el comportamiento y las acciones del vitoto.

[...] entonces se pide permiso, yo voy a sacar esto, si hay ahí cocos de monte, si hay otros bejucos, o sea itodo lo que hay!, entonces eso es lo que yo voy a reemplazar después ya con mi siembra, cuando usted va a sembrar, esos que se arrastran, esos que suben, entonces eso mismo es lo que uno reemplaza. (Gil Jairo, 2013).

El río se constituye en un escenario de aprendizaje cuyo origen se simboliza con la caída del árbol de la abundancia. (Diario de campo, Pérez, 2013). 
El cuidado de la vida humana se sustenta en el conocimiento del mundo, que se ha configurado históricamente y que le ha permitido prevalecer en el contexto selvático. Estas experiencias se entretejen y se renuevan en una cultura milenaria como la uitoto, que ha sabido interpretar los ciclos de la naturaleza y las condiciones climáticas, para poder armonizar las prácticas desde la maloca y el mambeadero, con rituales y ceremoniales como el baile del Yuak+, entre otros, al realizar la chagra, la pesca o cacería, procesos que también se referencian en los trabajos de Takacs (1996), Ulloa (2004) y Toledo y Barrera-Bassols (2008), y que reiteran el vínculo y la coexistencia con los demás seres desde lo que se podría considerar una ética y estética del mundo.

\section{Concepciones y prácticas de cuidado}

De acuerdo con las formas de concebir la biodiversidad desde lo cultural, es importante considerar la unidad entre la naturaleza-lo humano y lo espiritual, que vinculan los mitos en una estrecha relación entre el pensamiento-la palabra y la obra, es decir que el pensamiento se traduce en discurso y acciones que plantean una coherencia entre su ser individual y colectivo. Por lo cual, es desde una mirada integradora y compartida, que a continuación se presentan algunos de los aspectos que hacen parte de las prácticas de cuidado del vitoto:

\begin{tabular}{|c|c|c|}
\hline Componentes & Prácticas de cuidado & Consideraciones \\
\hline $\begin{array}{l}\text { Conocimientos: } \\
\text { sistema de } \\
\text { conocimiento }\end{array}$ & $\begin{array}{l}\text { Para nosotros realmente todo tiene vida, todo (Gil Jairo, } \\
2012 \text { ). } \\
\text { Cuando nosotros estamos pensando como indígenas y } \\
\text { que estamos como en riesgo de desaparecer, sí, porque } \\
\text { con todo lo de la globalización, pues nos meten a todos } \\
\text { allá entonces sucediendo esto, si usted no transmite ese } \\
\text { conocimiento, żque usted espera? (Fidelia, 2012) }\end{array}$ & $\begin{array}{l}\text { Conocimientos-Lo epistémico: Todo se encuentra Integrado. } \\
\text { Desde el mito de origen las prácticas epistémicas asociadas al } \\
\text { cuidado de la vida, se constituyen en prácticas de vida (mitos, } \\
\text { cantos, bailes). } \\
\text { Madre Tierra, Monifue. Especialidad del conocimiento: Bailes, } \\
\text { medicina, consejo, chamanismo. (Baile delYuak+) Formas de } \\
\text { organizar el cosmos y la naturaleza, desde el mito de origen } \\
\text { como explicación de la vida y su cuidado. }\end{array}$ \\
\hline $\begin{array}{l}\text { Educación } \\
\text { propia }\end{array}$ & $\begin{array}{l}\text { Se orienta la vida desde el mambeadero, a través de } \\
\text { los mitos de origen. Pues yo estoy tratando de seguir o } \\
\text { de aprender todo lo que tiene que ver con la parte de } \\
\text { medicina en la niñez (Gil Jairo.2012). } \\
\text { Si los padres no dietan y siguen el consejo del mayor, } \\
\text { antes que nazca el niño, esto se va a ver, porque el niño } \\
\text { va a ser rebelde, (Pérez, 2013. Diario de campo). } \\
\text { Como dice mi abuelo la educación para nosotros es de } \\
\text { por vida, la enseñanza es para todos, entonces, si a mí } \\
\text { me gusta la parte de medicina pues sobre eso yo voy a } \\
\text { seguir investigando, si al otro le gusto de cantar, de hacer } \\
\text { baile pues de eso va a seguir (Gil Jaira, 2012). }\end{array}$ & $\begin{array}{l}\text { El conocimiento del cuidado de la vida, se vive a través de } \\
\text { las prácticas de cuidado que vinculan los mitos y los rituales y } \\
\text { ceremoniales. Se educa en la vida y para la vida. El contexto } \\
\text { cultural determina los esquemas de significación transmitidos } \\
\text { en los escenarios de aprendizaje (maloca, río, selva y chagra). } \\
\text { La enseñanza de los mitos, los bailes y cantos comprenden } \\
\text { el orden cósmico, la diversidad de la vida, las funciones } \\
\text { e interacciones como un todo integrado, que articulan los } \\
\text { mundos tangible e intangible. }\end{array}$ \\
\hline Salud & $\begin{array}{l}\text { Para nosotros cada planta que se siembre cumple una } \\
\text { función en la alimentación de nosotros, por ejemplo, } \\
\text { el abuelo decía que hay unos frutos especiales que se } \\
\text { sembraban para que limpiaran la sangre, otro para que } \\
\text { mejorara la articulaciones cada uno tiene una función } \\
\text { (GilJairo, 2012). } \\
\text { Mi abuelo tenía esa virtud como de sanar, él preparaba } \\
\text { el yagé pero de la planta y él se iba y allá él empezaba a } \\
\text { narrar, o sea él dormido porque eso dice que se va a otro } \\
\text { mundo, entra es en oración, él lo narra o sea esa oración } \\
\text { para sanar, (Fidelia, 2012). }\end{array}$ & $\begin{array}{l}\text { La medicina ancestral se constituye en una práctica de } \\
\text { cuidado de la vida humana. Forma parte del conocimiento } \\
\text { empírico que se ha configurado históricamente y se actualiza. } \\
\text { Las propiedades de las plantas y la oración, posibilitan la } \\
\text { curación como vínculo entre materialidad y espiritualidad. Este } \\
\text { conocimiento se transmite al colectivo, acompañado de rituales } \\
\text { y ceremoniales. }\end{array}$ \\
\hline
\end{tabular}




\begin{tabular}{|c|c|c|}
\hline Componentes & Prácticas de cuidado & Consideraciones \\
\hline Alimento & $\begin{array}{l}\text { La selva, siempre está ahí y está surgiendo, porque la } \\
\text { madre tierra pues es la madre y pues, de ahí sale todo } \\
\text { y no tiene porque desaparecer de un momento a otro } \\
\text { a menos de que caiga un rayo o lo fumiguen o le haga } \\
\text { todo lo que el estado ha hecho. (Fidelia, 2012). } \\
\text { Para nosotros la tierra no es nuestra, nos la dieron para } \\
\text { nosotros, cuidarla (Francisco, 2D 12). } \\
\text { Hay que dejar para los que vienen, es tanto profe que } \\
\text { por ejemplo usted hace su chagra, usted siembra, porque } \\
\text { para nosotros la tierra no es nuestra (Fidelia, 2013). } \\
\text { Solamente bajo el buen uso de la palabra de vida, } \\
\text { conseguiremos lo que necesitamos para nuestros pueblos } \\
\text { y sus criaturas. Esto es algo que jamás debemos negar, al } \\
\text { contrario es un principio que tenemos que reproducir al } \\
\text { interior de nuestro corazón antes de sembrarlo en la tierra } \\
\text { (Azicatch, 2007, p.8). }\end{array}$ & $\begin{array}{l}\text { Representa un saber cuidar la vida y se concreta a través de } \\
\text { la chagra: Sembrar la vida, alimento material y espiritual. } \\
\text { El conocimiento de la dinámica de la selva y el río forman } \\
\text { parte del alimento a través de la caza y la pesca. Se extrae } \\
\text { únicamente para consumo, no se acumula. La orientación de } \\
\text { manejo se fundamenta en el calendario etnoecológico que } \\
\text { comprende un tiempo cíclico que interpreta el espacio local y } \\
\text { su regulación como se expresa en el calendario etnoecológico. }\end{array}$ \\
\hline Territorio & $\begin{array}{l}\text { Nosotros desde que nacemos estamos icuidando la vida!, } \\
\text { eso ya está como, o sea es ya un mandato, empezando } \\
\text { porque nosotros, lo hacemos de una forma hasta } \\
\text { inconsciente yo digo (Fidelia, 2013). } \\
\text { Después que ya uno es mambeador o que uno está en } \\
\text { esa preparación pues por eso le recomiendan a uno pues } \\
\text { presentarse todas las noches, yo creo que ahí el dialogo } \\
\text { que uno realiza es precisamente para uno mirar todo el } \\
\text { trabajo del mañana, la cacería, o si voy a trabajar la casa } \\
\text { o si voy a sacar lefia, bueno, esa noche usted organiza, } \\
\text { bueno voy a hacer esto mañana, ahí organiza esa parte } \\
\text { y entonces en ese momento si va a hacer chagra usted } \\
\text { está pidiendo el permiso, si, es en esa medida, no entrar } \\
\text { directo sino siempre pedir permiso (Gil Jairo, 2012). }\end{array}$ & $\begin{array}{l}\text { El territorio para convivir y saber habitar: comprende relaciones } \\
\text { de convivencia cósmica como un todo interrelacionado. } \\
\text { Implica Armonización de las relaciones entre la Naturaleza- lo } \\
\text { humano y lo espiritual. La Tierra genera vida y se constituye } \\
\text { en un lugar de habitación (nunca completo, ni definitivo), sin } \\
\text { pretensiones de apropiación. La otredad emerge al reconocer } \\
\text { la naturaleza como un sujeto, en las prácticas de cuidado, } \\
\text { se dialoga para pedir permiso a los dueños espirituales al } \\
\text { realizar chagra, pesca o caza. Se regula la vida: A través de } \\
\text { las prácticas de cuidado de la vida. Para el uitoto laDieta es } \\
\text { un saber comportarse con la naturaleza y el colectivo, desde la } \\
\text { ley de vida. }\end{array}$ \\
\hline
\end{tabular}

Figura 6. Prácticas de cuidado del pueblo vitoto

Fuente: elaboración propia.

La relación perfecta está entre lo divino lo humano y la naturaleza y para nosotros de manera directa yo dijera de relación de igual a igual, hombre igual a naturaleza, naturaleza igual a lo divino o viceversa. (Farekatde, 2013, sabedor vitoto-muruy).

Durante los diferentes diálogos y recorridos, aparecen expresiones sobre el cuidado de la vida y el cuidado del conocimiento, las cuales guardan una profunda relación con la ley de vida, fundamento del ethos vitoto-muruy. El cuidado se vislumbra como un fenómeno para la consciencia, que se lleva a la experiencia, y toma forma en las prácticas de los sujetos (Boff, 2002, p. 171), tanto en el plano individual como en el colectivo. En este sentido, hablar del cuidado no es algo independiente del sujeto, por el contrario implica una dimensión ontológica que entra a formar parte de la constitución del ser humano.

digamos en este calendario hay unos tiempos, dependiendo del tiempo o sea ya uno sabe que eso hay que hablarse, entonces el tema de fondo ya es el tiempo, estamos en creciente, estamos en subienda de pescado, estamos en tiempo de enfermedad, estamos en tiempo de verano. (Gil Jairo, 2013).

La complejidad del conocimiento ecológico ancestral de la etnia vitoto-muruy condensado en el calendario etnoecológico, como vía para plasmar las dinámicas de factores climáticos, en especial, las épocas de lluvias (aumento del caudal de los ríos y zonas de inundación), así como las épocas de verano, articulado 
con los procesos de producción de frutos, y la asociación con la fauna del lugar, permiten orientar las prácticas culturales en relación con la apertura de chagra, siembra y recolección, así como los momentos y lugares para la pesca y cacería. Todo se encuentra integrado tanto en lo material como en lo espiritual, donde también está presente el monifue o abundancia que expresa la producción de vida y su cuidado.

La Madre Tierra orienta el cuidado de la vida desde una relacionalidad con los demás existentes, y con un sentido de cuidado y de reciprocidad de los seres. El monifue o abundancia representa la palabra, el conocimiento del cuidado de un "ver crecer a su gente" que se advierte en el conocimiento y las prácticas de cuidado de la vida humana (bailes, medicina, consejo, chamanismo), que apela a todo lo que el ser humano emprende, proyecta y hace (Heidegger, 1927), en tal sentido, se hace especial énfasis sobre el cuidado, al orientar el pensamiento, la palabra y la obra en la cotidianidad del individuo y del colectivo clánico. El baile del Yuak + fortalece la memoria biocultural que plantea un presente que se teje con el tiempo mítico y se ocupa de armonizar las relaciones entre la naturaleza-lo humano y lo espiritual, es decir, cuando la existencia del otro también tiene importancia para sí, como una actitud de cuidado hacia los demás y que propicia un sentido de responsabilidad del vivir juntos y un saber habitar el territorio.

\section{Conclusiones}

Las concepciones de biodiversidad como nuevo énfasis de la naturaleza, permiten comprender su complejidad a través de las narrativas y explicaciones de los docentes en formación inicial de la Licenciatura en Biología, tomando en consideración el enfoque semiótico de la cultura (Geertz, 2004) y lo cultural en términos adjetivos (García Canclini, 2004). Esta mirada desde lo ontológico, en términos culturales, no uniformiza sino, por el contrario, se constituye en expresión de la diferencia, que no solo tiene lugar entre culturas sino dentro de ellas, de modo que, si bien se habla de una unidad entre la naturaleza-lo humano y lo espiritual, de acuerdo con su cosmovisión, también se dinamiza y se configuran nuevas formas de apropiación y de enunciación.

La construcción del mundo desde la palabra permite considerar su afirmación de la vida, que pretende una coherencia entre el pensamiento-la palabra y la obra, referente desde el cual se alienta una serie de articulaciones, como formas de mantener la unidad y la palabra no desde una mirada esencialista de la existencia, sino desde una relacionalidad dinámica que opera bajo la idea del cuidado de la vida.

Para la cultura vitoto, el cuidado del conocimiento se erige como principio para el cuidado de la vida, a través del cual, se construye una manera de ser y de habitar el mundo, que dota de sentido la existencia del vitoto-muruy, constituyéndose en uno de los pilares de estas concepciones. Así, para el vitoto, el conocimiento del mundo es situado y se fundamenta en el cuidado de la vida como filosofía de existencia, que permite a sus miembros desarrollar diferentes acciones, bajo un conjunto de normas y valores evaluados en función de los fines que se han configurado en la etnia uitoto-muruy, como formas de constitución del conocimiento soportadas en las experiencias que se dinamizan desde lo colectivo.

La ética del cuidado de la vida, en sus diversas manifestaciones, se constituye en un compromiso innegociable al aludir al respeto y la reciprocidad, que no se restringe a las interrelaciones humanas, sino que se despliega a cada tipo de interacción, sea entre individuos y grupos humanos, entre el ser humano 
y la naturaleza o entre el ser humano y lo espiritual como también lo expresa Estermann (1998), aspecto que forma parte de sus discursos y prácticas desde un saber habitar el mundo.

En síntesis, es importante reconocer que la educación en ciencias y la formación de profesores, requiere de una propuesta que contemple la complejidad de la configuración de las concepciones desde perspectivas culturales, así como la oportunidad para pensar en una formación de profesores de ciencias sensible a los contextos culturales, la diversidad y la diferencia (Molina et al., 2014; Yuen, 2010). En tal sentido, se requiere ampliar la mirada frente a otras formas de conocimiento que han aportado en la producción de la vida y su cuidado, desde tiempos milenarios y que permiten incluir a Colombia como uno de los países más biodiversos del planeta, lo cual debe tener una mayor visibilidad en los procesos educativos, posibilitar el tejido de puentes entre conocimientos, con una educación más centrada en las realidades del país.

\section{Referencias}

Azicatch. (2007). Plan de vida de los hijos del tabaco, la coca y la yuca dulce y plan de abundancia de La Chorrera. Chorrera-Amazonas: Asociación de Cabildos y Autoridades Tradicionales de La Chorrera.

Boff, L. (2002). El cuidado esencial. Ética de lo humano. Compasión por la tierra. Madrid: Trotta.

Bravo, M. (2015). Pensamiento, palabra, acción y territorio: perspectivas y desafíos para el abordaje de cuestiones ambientales y territoriales, con pobladores de la cuenca del bajo río Caquetá-Colombia. Tecné, Episteme y Didaxis, 38, 75-94.

Bruner, J. (1984). Acción, pensamiento y lenguaje. Madrid: Alianza.

Bruner, J. (1988). Realidad mental y mundos posibles. B. López (trad.). Barcelona Gedisa.

Bruner, J. (2006). Actos de significado: más allá de la revolución cognitiva. Madrid: Alianza.

Convenio sobre la Diversidad Biológica. (1992). Texto del Convenio. Recuperado de http://www.cbd.int/ convention/text/

Descola, P. (2012). Más allá de la naturaleza y cultura. Buenos Aires; Madrid: Amorrortu.

Escobar, A. (1999). Whose knowledge, whose nature? Biodiversity conservation and social movements' political ecology. Journal of Political Ecology, 5(1), 53-82.

Escobar, A. (201 1). Epistemologías de la naturaleza y colonialidad de la naturaleza. Variedades de realismo y constructivismo. En L. Montenegro Martínez (ed.), Cultura y naturaleza, 1. a ed. Bogotá: Jardín Botánico de Bogotá, José Celestino Mutis. 
Estermann, J. (1998). Filosofía andina. Estudio Intercultural de la sabiduría autóctona andina. Quito: Abya-Yala.

García Canclini, N. (2004). Diferentes, desiguales y desconectados. Mapas de la interculturalidad. Barcelona: Gedisa.

Geertz, C. (1987). La interpretación de las culturas. México: Gedisa.

Geertz, C. (1996). Los usos de la diversidad. Barcelona: Paidós.

Ghilarov, A. (1996). What does "biodiversity" means: scientific problem or convenient myth? Trends in Ecology and Evolution, 17 (7), 304-306.

Heidegger, M. (1927). El ser y el tiempo. Recuperado de www.philosophia.cl /

Jeffries, M. (1997). Biodiversity and conservation. Londres: Routledge.

Lovejoy, T. E. (1980). The global 2000 report to the president, vol. 2 (The Technical Report, pp. 327-332) (Barney, Go., ed.).

Maffi, L. (2005). Linguistic, cultural, and biological diversity. The Annual Review of Anthropology 29, 599-617

Melo, N. (2017). Los puentes en la enseñanza de las ciencias: un compromiso para comprender las investigaciones sobre las relaciones entre conocimientos científicos escolares y conocimientos ecológicos tradicionales. Tecné, Episteme y Didaxis, 42, 43-61.

Molina, A. (2005). El "otro" en la constitución de identidades culturales. En C. Piedrahita y E. Paredes (eds.), Cultura política, identidades y nueva ciudadanía, vol. 2. (pp. 141-173). Cúcuta: Sic.

Molina, A. (2012). Una visión crítica de la enseñanza de las ciencias: conversando con la profesora Sandra sobre la diversidad cultural y sus perspectivas educativas. Educación y Ciudad (23), 133-150.
Molina, A., Mosquera, C., Utges, G., Mojica, L., Cifuentes, M., Reyes D., Martínez, A. y Pedreros, R. (2014). Concepciones de los profesores sobre el fenómeno de la diversidad cultural y sus implicaciones en la enseñanza de las ciencias. Bogotá: Universidad Distrital Francisco José de Caldas.

Montoya, S. (1994). Mitología del encuentro y del desencuentro. Bogotá: Universidad Nacional de Colombia.

Olivé, L. (2009). Por una auténtica interculturalidad basada en el reconocimiento de la pluralidad epistemológica. En E. Sader (ed.), Pluralismo epistemológico. La Paz: Clacso; Organización de las Naciones Unidas (ONU) (1992).

Palacio, G. (2001). Naturaleza en disputa. Ensayos de historia ambiental en Colombia 1850-1995. Bogotá: Unibiblos.

Pérez, M. R. (2013b). Concepciones de biodiversidad: una mirada desde la diversidad cultural. Magis, Revista Internacional de Investigación en Educación, 6(12), 133-151.

Pitrou, P., Valverde, M. y Neurath, J. (coords). (201 1). La noción de vida en Mesoamérica. México: Universidad Nacional Autónoma de México; Instituto de Investigaciones $\mathrm{Fi}$ lológicas; Centro de Estudios Mexicanos y Centroamericanos.

Plantin, C. (2014). Lengua, argumentación y aprendizajes escolares. Tecné, Episteme y Didaxis, (36), 95-114.

Ramírez, C. (2005). La perspectiva de los wayuu sobre los conocimientos y la biodiversidad. Pueblo wayuu, La Guajira, Colombia. En E. Escobar, P. Escobar, A. Pazmiño y A. Ulloa (eds.), Las mujeres indígenas en los escenarios de la biodiversidad. Bogotá: UICN, Fundación Natura Colombia, ICANH. 
Rozo, J. (2005). Una mirada al pensamiento vitoto. Al Margen, 14, 78-84.

Sarkar, S. (2005). Biodiversity and environmental philosophy. Nueva York: Cambridge University Press.

Sarkar, S. (2008). Biodiversity and environmental philosophy. Nueva York: Cambridge University Press.

Solbrig, O. T. (1991). The origin and function of biodiversity. Environment Science and Policy for Sustainable Development, 33, 16-38.

Takacs, D. (1996). The idea of biodiversity: Philosophy of paradise. Baltimore: The Johns Hopkins University Press.

Toledo, V. M. y Barrera-Bassols, N. (2008). Memoria biocultural. La importancia ecológica de las sabidurías tradicionales. Barcelona: Icaria.

Ulloa, A. (2004). La construcción del nativo ecológico. Bogotá: ICANH.

Ulloa, A. (2011). Concepciones de la naturaleza en la antropología actual. En L. Montenegro (ed.), Cultura y naturaleza, 1. ${ }^{a}$ ed. Bogotá: Jardín Botánico de Bogotá, José Celestino Mutis.

Urbina, F. (2010). Las palabras del origen. Breve compendio de la mitología de los vitotos. Bogotá: Ministerio de Cultura.

Vasilachis, I. (2006). Estrategias de investigación cualitativa. Barcelona: Gedisa.

Yuen, C. (2010). Dimensions of diversity: Challenges to secondary school teachers with implications for intercultural teacher education. Teaching and Teacher Education, 26(3), 732-774.

\section{Para citar este artículo}

Pérez, R. (2019). Concepciones de biodiversidad y prácticas de cuidado de la vida desde una perspectiva cultural. Reflexiones a propósito de la formación de profesores de biología. Tecné, Episteme y Didaxis: TED, 45, 17-34. 